\title{
Memories of Raoul Bott (Commentary on [96])
}

\author{
András Szenes
}

Raoul Bott was a wonderful lecturer, with a talent to get to the essence of the matter, masterfully evading most technical complications. For the uninitiated, this often had the effect of creating the illusion of understanding everything. This understanding then painfully disappeared the next morning, replaced by a slew of questions, and this is where the actual learning from the talk began.

One of his favorite anecdotes was the story of his first talk at the IAS in Princeton. A week before the talk, Bott came to his mentor, Marsden Morse, to present what he was planning to say during his talk. Morse seemed to be quite content, but said that that was too much: "Cut it in half!" Bott took on the task with pleasure, and he returned to Morse with a version, where he thought to have kept only the absolutely necessary parts. To his bewilderment, Morse had again the same reaction: he thought the talk was well thought out, the only necessary modification was to "cut it one more time in half."

Another stylistic remark Bott often repeated was never to give the outline of the talk at the start. For him this was partly philosophical: he thought of a talk as telling a story; but it also in part practical: "they will never get to the end of it"-he would say.

Bott loved calculations, and he was rather proud of the description of the cohomology of the moduli space of stable bundles of rank 2 on a genus-2 Riemann surface that one can find in his Yang-Mills paper with Atiyah. He said it took him an entire summer to derive this structure from the relations introduced by David Mumford.

He was all the more incredulous, when the formula of Verlinde appeared in 1988-89, implicitly describing intersection numbers on the moduli space for any genus and rank. I became his student exactly at this time; I remember Bott being excited and agitated. In fact, my first assignment was to disprove the formula. When all my efforts failed, we began calculating in the Fusion algebra, and he was rather pleased with the "discrete integration" interpretation of the formula, and the analogies with the representation rings of finite groups.

He was in permanent competition with the physicists, in a competition that he was, quite often, all too happy to lose.

\footnotetext{
A. Szenes $(\bowtie)$

Section de Mathématiques, Université de Genève, Geneva, Switzerland

e-mail: andras.szenes@unige.ch
} 